\title{
TRUNCATION ERROR BOUNDS FOR BRANCHED CONTINUED FRACTION WHOSE PARTIAL DENOMINATORS ARE EQUAL TO UNITY
}

T. M. Antonova, R. I. Dmytryshyn. Truncation error bounds for branched continued fraction whose partial denominators are equal to unity, Mat. Stud. 54 (2020), 3-14.

The paper deals with the problem of obtaining error bounds for branched continued fraction of the form

$$
\sum_{i_{1}=1}^{N} \frac{a_{i(1)}}{1}+\sum_{i_{2}=1}^{i_{1}} \frac{a_{i(2)}}{1}+\sum_{i_{3}=1}^{i_{2}} \frac{a_{i(3)}}{1}+\cdots
$$

which is the multidimensional generalization of a corresponding continued fraction. Here the elements are complex numbers. This problem is complicated by the fact that the methods for truncation-error analysis of continued fractions are generally not transferred to branched continued fractions.

By means of fundamental inequalities method the truncation error bounds are obtained for the above mentioned branched continued fraction providing its elements belong to some rectangular sets of a complex plane. An important component of this method is the estimations of the so-called 'tails' of branched continued fraction. The established conditions for the elements depend on two sequences of real numbers. By choosing certain possible values of these sequences, we have established two simple and constructive criteria for deriving error bounds for branched continued fraction.

Applications are considered for several classes of branched continued fraction expansions including the multidimensional $S$-, $A-, J$-fractions with independent variables. These functional branched continued fractions are an efficient tool for the approximation of analytic functions of several complex variables, which are represented by multiple power series.

1. Introduction. Let $N$ be a fixed integer number, $i(k)=\left(i_{1}, i_{2}, \ldots, i_{k}\right)$ be a multiindex and let $\mathcal{I}=\left\{i(k): 1 \leq i_{p} \leq i_{p-1}, 1 \leq p \leq k, i_{0}=N, k \in \mathbb{N}\right\}$ be a set of multiindices.

In the paper, we study the convergence of a branched continued fraction (hereafter abbreviated as BCF)

$$
\sum_{i_{1}=1}^{N} \frac{a_{i(1)}}{1}+\sum_{i_{2}=1}^{i_{1}} \frac{a_{i(2)}}{1}+\sum_{i_{3}=1}^{i_{2}} \frac{a_{i(3)}}{1}+\cdots
$$

where $a_{i(k)}, i(k) \in \mathcal{I}$, are complex numbers.

The expression

$$
f_{n}=\sum_{i_{1}=1}^{N} \frac{a_{i(1)}}{1}+\sum_{i_{2}=1}^{i_{1}} \frac{a_{i(2)}}{1}+\cdots+\sum_{i_{n}=1}^{i_{n-1}} \frac{a_{i(n)}}{1}
$$

2010 Mathematics Subject Classification: 11J70, 32A17, 41A25.

Keywords: convergence; truncation error; branched continued fraction. doi:10.30970/ms.54.1.3-14 
is called the $n$-th approximant of (1), $n \geq 1$. The BCF (1) is said to converge if its sequence of approximants converges, and $\lim _{n \rightarrow \infty} f_{n}$ is called its value.

Many methods for proving the convergence of continued fractions and their generalization $\mathrm{BCF}$ are methods for proving the existence of limits of sequences of their approximants and, therefore, they do not give truncation error bounds (see, e.g., [3, 7, 9, 15, 17]). However, these estimates are important for applying them to the approximation of functions of one or several complex variables. Methods for truncation-error analysis of continued fractions are presented in [21]. Unfortunately, in general, the above mentioned methods are not transferable to BCF.

Among the few papers on the above, we noteworthy the papers [8, 10], where, using the known truncation error bounds for a continued fraction by the method of induction by dimension of $\mathrm{BCF}$, the truncation error bounds are established for the $\mathrm{BCF}$

$$
\sum_{i_{1}=1}^{N} \frac{1}{b_{i(1)}}+\sum_{i_{2}=1}^{i_{1}} \frac{1}{b_{i(2)}}+\sum_{i_{3}=1}^{i_{2}} \frac{1}{b_{i(3)}}+\cdots
$$

where $b_{i(k)}, i(k) \in \mathcal{I}$, are constants belonging to angular sets of the complex plane. Also, we should like to mention the paper [13] in which obtained error bounds for the periodic BCF

$$
\frac{1}{1}+\sum_{i_{1}=1}^{N} \frac{c_{i_{1}}}{1}+\sum_{i_{2}=1}^{i_{1}} \frac{c_{i_{2}}}{1}+\sum_{i_{3}=1}^{i_{2}} \frac{c_{i_{3}}}{1}+\cdots
$$

under the following conditions: if the first element belongs to the complex plain with the cut $(-8 ;-1 / 4]$ and the sum of moduli of other elements is bounded by a certain number; if elements belong to their corresponding parabolic regions or union of that parabolic regions and all their moduli, except of the first one, are bounded.

The paper is a continuation of work [4], which is related with research initiated in [1, 2]. To study convergence of (1) we use the fundamental inequalities method (see $[1,2,5,6,11]$ ). An important component of the above mentioned method is the estimation of the so-called 'tails', which for the BCF (1) can be determined as follows:

$$
G_{i(k)}^{(n)}=1+\sum_{i_{k+1}=1}^{i_{k}} \frac{a_{i(k+1)}}{G_{i(k+1)}^{(n)}}, i(k) \in \mathcal{I}, 1 \leq k \leq n-1, n \geq 2,
$$

with the initial conditions $G_{i(s)}^{(s)}=1, i(s) \in \mathcal{I}, s \geq 1$. We assume that the fundamental inequalities are satisfied for the $\mathrm{BCF}(1)$ if

$$
G_{i(k)}^{(s)} \neq 0 \text { for all } i(k) \in \mathcal{I}, 1 \leq k \leq s, s \in \mathbb{N}
$$

and there exist positive numbers $M$ and $\rho_{l}, l \geq 1$, such that for all $s \geq 1, n \geq 2$ and $1 \leq k \leq n-1$ the following inequalities hold

$$
\left|a_{i(1)}\right| \leq M\left|G_{i(1)}^{(s)}\right|, 1 \leq i_{1} \leq N,\left|a_{i(k+1)}\right| \leq \rho_{k}\left|G_{i(k)}^{(n)} G_{i(k+1)}^{(n)}\right|, i(k) \in \mathcal{I}, 1 \leq i_{k+1} \leq i_{k} .
$$

For use in the proof of our results, we include the following theorem that is proved in [1].

Theorem 1. Let the elements of the BCF (1) satisfy the inequalities (3), (4) and

$$
C_{N+n}^{N-1} \prod_{l=1}^{n} \rho_{l} \rightarrow 0 \text { as } n \rightarrow \infty \text {. }
$$


Then the BCF (1) converges to a finite value $f$, and if $f_{n}$ denotes the $n$-th approximant, then

$$
\left|f-f_{n}\right| \leq M C_{N+n}^{N-1} \prod_{l=1}^{n} \rho_{l}, n \geq 1 .
$$

We remark that $(N-1) !\left(\sum_{n=1}^{\infty} \varrho^{n+N}\right)^{(N-1)}=\sum_{n=1}^{\infty} \varrho^{n+1} C_{N+n}^{N-1}$ for $0 \leq \varrho<1$. In particular, this implies $\varrho^{n} C_{N+n}^{N-1} \rightarrow 0(n \rightarrow+\infty)$ for $0 \leq \varrho<1$. Thus in the case $0 \leq \varrho_{l} \leq \varrho<1$ $(\forall l \geq 1)$ we have

$$
0 \leq C_{N+n}^{N-1} \prod_{l=1}^{n} \varrho_{l} \leq \varrho^{n+1} C_{N+n}^{N-1} \rightarrow o, \quad(n \rightarrow+\infty) .
$$

Therefore condition (5) is satisfied.

2. Main results. Let $\left\{t_{i(k)}\right\}_{i(k) \in \mathcal{I}, k \geq 2}$ and $\left\{\alpha_{i(k)}\right\}_{i(k) \in \mathcal{I}}$ be sequences of real numbers. We set for $i(k) \in \mathcal{I}$

$$
\begin{gathered}
x_{i(k)}=\operatorname{Re}\left(a_{i(k)} e^{i\left(\alpha_{i(k-1)}+\alpha_{i(k)}\right)}\right), y_{i(k)}=\operatorname{Im}\left(a_{i(k)} e^{i\left(\alpha_{i(k-1)}+\alpha_{i(k)}\right)}\right), k \geq 2 ; \\
u_{i(k)}^{(n)}=\operatorname{Re}\left(G_{i(k)}^{(n)} e^{i \alpha_{i(k)}}\right), v_{i(k)}^{(n)}=\operatorname{Im}\left(G_{i(k)}^{(n)} e^{\left.i \alpha_{i(k)}\right)}, 1 \leq k \leq n, n \in \mathbb{N} ;\right. \\
T_{i(k)}=\sum_{i_{k+1}=1}^{i_{k}} t_{i(k+1)} ; \\
\mu_{i(k)}=\max \left\{\left(\cos ^{2} \alpha_{i(k)}+\left(1-T_{i(k)}\right)^{2}\right)^{1 / 2} \sin \alpha_{i(k)}, 1-T_{i(k)},\left(1-T_{i(k)} / 2\right) \sin 2 \alpha_{i(k)}\right\} .
\end{gathered}
$$

Next, let $i(k-1)$ be an arbitrary fixed multiindex from $\mathcal{I}, k \geq 2$, and let among the values $x_{i(k)}, 1 \leq i_{k} \leq i_{k-1}$, there are $n_{i(k-1)}$ nonnegative numbers, $0 \leq n_{i(k-1)} \leq i_{k-1}$. We define the sets $\mathcal{I}_{i(k-1)}^{(1)}$ and $\mathcal{I}_{i(k-1)}^{(2)}$ as

$$
\mathcal{I}_{i(k-1)}^{(1)}=\left\{i_{k}: i(k) \in \mathcal{I}, x_{i(k)} \geq 0\right\}, \mathcal{I}_{i(k-1)}^{(2)}=\left\{i_{k}: i(k) \in \mathcal{I}, x_{i(k)}<0\right\} .
$$

Then $\mathcal{I}_{i(k-1)}^{(1)} \cup \mathcal{I}_{i(k-1)}^{(2)}=\left\{1,2, \ldots, i_{k-1}\right\},\left|\mathcal{I}_{i(k-1)}^{(1)}\right|=n_{i(k-1)}$ and $\left|\mathcal{I}_{i(k-1)}^{(2)}\right|=i_{k-1}-n_{i(k-1)}$.

We prove the following lemma.

Lemma 1. Let the elements of the BCF (1) satisfy the inequalities

$$
-t_{i(k)} \mu_{i(k)} \cos \alpha_{i(k-1)} \leq x_{i(k)} \leq t_{i(k)} \mu_{i(k)} \sin \alpha_{i(k-1)}, y_{i(k)} \geq 0 \text { for all } i(k) \in \mathcal{I}, k \geq 2,
$$

where $x_{i(k)}, y_{i(k)}$ and $\mu_{i(k)}, i(k) \in \mathcal{I}, k \geq 2$, are defined by (6) and (9), respectively; $t_{i(k)}$, $k \geq 2$, and $\alpha_{i(k)}, i(k) \in \mathcal{I}$, are real numbers such that

$$
t_{i(k)}>0, k \geq 2, T_{i(k)} \leq 1,0<\alpha_{i(k)}<\pi / 2, i(k) \in \mathcal{I},
$$

where $T_{i(k)}, i(k) \in \mathcal{I}$, are defined by (8). Then for all $i(k) \in \mathcal{I}, 1 \leq k \leq n$, and $n \in \mathbb{N}$ the following inequalities are valid

$$
u_{i(k)}^{(n)} \geq\left(1-T_{i(k)}^{(2)}\right) \cos \alpha_{i(k)}, v_{i(k)}^{(n)} \geq\left(1-T_{i(k)}^{(1)}\right) \sin \alpha_{i(k)} \text { and }\left|G_{i(k)}^{(n)}\right| \geq \mu_{i(k)}>0,
$$

where $G_{i(k)}^{(n)}$ and $u_{i(k)}^{(n)}, v_{i(k)}^{(n)}, i(k) \in \mathcal{I}, 1 \leq k \leq n, n \in \mathbb{N}$, are defined by (2) and (7), respectively; $T_{i(k)}^{(r)}=\sum_{i_{k+1} \in \mathcal{I}_{i(k)}^{(r)}} t_{i(k+1)}, \mathcal{I}_{i(k)}^{(r)}$ is defined in $(10)$ and $i(k) \in \mathcal{I}, r \in 1,2$. 
Proof. Let $n$ be an arbitrary integer number $n$. Using relations (2) and (7), by induction on $k$ we show that for each multiindex $i(k) \in \mathcal{I}, 1 \leq k \leq n$ the inequalities (13) are valid.

For $k=n$ and for all $i(n) \in \mathcal{I}$ relations (13) are obvious. By induction hypothesis we assume that (13) hold for $k=p$ and for all $i(p) \in \mathcal{I}$ such that $p \leq n$. Then, use of inequalities (11) and (12) for $k=p-1$ and an arbitrary multiindex $i(p-1) \in \mathcal{I}$ leads to

$$
\begin{gathered}
u_{i(p-1)}^{(n)} \geq \cos \alpha_{i(p-1)}+\sum_{i_{p} \in \mathcal{I}_{i(p-1)}^{(2)}} \frac{x_{i(p)} u_{i(p)}^{(n)}}{\left|G_{i(p)}^{(n)}\right|^{2}} \geq\left(1-\sum_{i_{p} \in \mathcal{I}_{i(p-1)}^{(2)}} \frac{t_{i(p)} \mu_{i(p)}}{\left|G_{i(p)}^{(n)}\right|}\right) \cos \alpha_{i(p-1)} \geq \\
\geq\left(1-T_{i(p-1)}^{(2)}\right) \cos \alpha_{i(p-1)}, \\
v_{i(p-1)}^{(n)} \geq \sin \alpha_{i(p-1)}-\sum_{i_{p} \in \mathcal{I}_{i(p-1)}^{(1)}} \frac{x_{i(p)} v_{i(p)}^{(n)}}{\left|G_{i(p)}^{(n)}\right|^{2}} \geq\left(1-T_{i(p-1)}^{(1)}\right) \sin \alpha_{i(p-1)} .
\end{gathered}
$$

Let us estimate the value $\left|G_{i(p-1)}^{(n)}\right|$. We have

$$
\begin{gathered}
\left|G_{i(p-1)}^{(n)}\right|^{2} \geq \cos ^{2} \alpha_{i(p-1)}\left(1-T_{i(p-1)}^{(2)}\right)^{2}+\sin ^{2} \alpha_{i(p-1)}\left(1-T_{i(p-1)}^{(1)}\right)^{2}= \\
=\left(1-T_{i(p-1)}^{(2)}\right)^{2}\left(1+\cos 2 \alpha_{i(p-1)}\right) / 2+\left(1-T_{i(p-1)}^{(1)}\right)^{2}\left(1-\cos 2 \alpha_{i(p-1)}\right) / 2 .
\end{gathered}
$$

Let

$$
A_{i(p-1)}=\left(1-T_{i(p-1)}^{(1)}\right)^{2}+\left(1-T_{i(p-1)}^{(2)}\right)^{2} \text { and } B_{i(p-1)}=\left(1-T_{i(p-1)}^{(2)}\right)^{2}-\left(1-T_{i(p-1)}^{(1)}\right)^{2} .
$$

Since

$$
\begin{gathered}
A_{i(p-1)}=\left(1-T_{i(p-1)}+T_{i(p-1)}^{(2)}\right)^{2}+\left(1-T_{i(p-1)}^{(2)}\right)^{2}= \\
=1-2 T_{i(p-1)}^{(2)}+2\left(T_{i(p-1)}^{(2)}\right)^{2}+\left(1-T_{i(p-1)}\right)^{2}+2\left(1-T_{i(p-1)}\right) T_{i(p-1)}^{(2)}, \\
B_{i(p-1)}=\left(1-T_{i(p-1)}^{(2)}\right)^{2}-\left(1-T_{i(p-1)}+T_{i(p-1)}^{(2)}\right)^{2}= \\
=1-2 T_{i(p-1)}^{(2)}-\left(1-T_{i(p-1)}\right)^{2}-2\left(1-T_{i(p-1)}\right) T_{i(p-1)}^{(2)},
\end{gathered}
$$

then we have

$$
\begin{aligned}
& A_{i(p-1)}+B_{i(p-1)} \cos 2 \alpha_{i(p-1)}=1+\cos 2 \alpha_{i(p-1)}-2 T_{i(p-1)}^{(2)}\left(1+\cos 2 \alpha_{i(p-1)}\right)+2\left(T_{i(p-1)}^{(2)}\right)^{2}+ \\
& +\left(1-T_{i(p-1)}\right)^{2}\left(1-\cos 2 \alpha_{i(p-1)}\right)+2\left(1-T_{i(p-1)}\right) T_{i(p)}^{(2)}\left(1-\cos 2 \alpha_{i(p-1)}\right) \geq 1+\cos 2 \alpha_{i(p-1)}+ \\
& \quad+\left(1-T_{i(p-1)}\right)^{2}\left(1-\cos 2 \alpha_{i(p-1)}\right)-\left(1+\cos 2 \alpha_{i(p-1)}\right)^{2} / 2-2 T_{i(p-1)}^{(2)}\left(1+\cos 2 \alpha_{i(p-1)}\right)+ \\
& +2\left(T_{i(p-1)}^{(2)}\right)^{2}+\left(1+\cos 2 \alpha_{i(p-1)}\right)^{2} / 2 \geq\left(1-\cos ^{2} 2 \alpha_{i(p-1)}\right) / 2+\left(1-T_{i(p-1)}\right)^{2}\left(1-\cos 2 \alpha_{i(p-1)}\right) .
\end{aligned}
$$

Thus $\left|G_{i(p-1)}^{(n)}\right| \geq\left(\cos ^{2} \alpha_{i(p-1)}+\left(1-T_{i(p-1)}\right)^{2}\right)^{1 / 2} \sin \alpha_{i(p-1)}$.

Other elementary identity transformations yield

$$
\begin{gathered}
A_{i(p-1)}=1+\left(1-T_{i(p-1)}\right)^{2}+2\left(T_{i(p-1)}^{(2)}\right)^{2}-2 T_{i(p-1)} T_{i(p-1)}^{(2)}, \\
B_{i(p-1)}=1-\left(1-T_{i(p-1)}\right)^{2}+2\left(T_{i(p-1)}-2\right) T_{i(p-1)}^{(2)},
\end{gathered}
$$


and therefore

$$
\begin{gathered}
A_{i(p-1)}+B_{i(p-1)} \cos 2 \alpha_{i(p-1)}=1+\cos 2 \alpha_{i(p)}+\left(1-T_{i(p-1)}\right)^{2}\left(1-\cos 2 \alpha_{i(p-1)}\right)+2\left(T_{i(p-1)}^{(2)}\right)^{2}- \\
-2 T_{i(p-1)}^{(2)}\left(T_{i(p-1)}+\left(2-T_{i(p-1)}\right) \cos 2 \alpha_{i(p-1)}\right)=1+\left(1-T_{i(p-1)}\right)^{2}\left(1-\cos 2 \alpha_{i(p-1)}\right)+ \\
+\cos 2 \alpha_{i(p-1)}-\left(T_{i(p-1)}+\left(2-T_{i(p-1)}\right) \cos 2 \alpha_{i(p-1)}\right)^{2} / 2+ \\
+2\left(T_{i(p-1)}^{(2)}-\left(T_{i(p-1)}+\left(2-T_{i(p-1)}\right) \cos 2 \alpha_{i(p-1)}\right) / 2\right)^{2} \geq 1+\left(1-T_{i(p-1)}\right)^{2}\left(1-\cos 2 \alpha_{i(p-1)}\right)+ \\
+\cos 2 \alpha_{i(p-1)}-\left(T_{i(p-1)}+\left(2-T_{i(p-1)}\right) \cos 2 \alpha_{i(p-1)}\right)^{2} / 2=1+\left(1-T_{i(p-1)}\right)^{2}- \\
-2\left(1-T_{i(p-1)} / 2\right)^{2} \cos ^{2} 2 \alpha_{i(p-1)}-\left(T_{i(p-1)}\right)^{2} / 2=2-2 T_{i(p-1)}- \\
-2\left(1-T_{i(p-1)} / 2\right)^{2} \cos ^{2} 2 \alpha_{i(p-1)}+\left(T_{i(p-1)}\right)^{2} / 2=2\left(1-T_{i(p-1)} / 2\right)^{2} \sin ^{2} 2 \alpha_{i(p-1)} .
\end{gathered}
$$

It follows that $\left|G_{i(p-1)}^{(n)}\right| \geq\left(1-T_{i(p-1)} / 2\right) \sin 2 \alpha_{i(p-1)}$.

In addition, we have

$$
\left|G_{i(p-1)}^{(n)}\right|^{2} \geq\left(1-T_{i(p-1)}^{(2)}\right)^{2} \cos ^{2} \alpha_{i(p-1)}+\left(1-T_{i(p-1)}^{(1)}\right)^{2} \sin ^{2} \alpha_{i(p-1)} \geq\left(1-T_{i(p-1)}\right)^{2},
$$

that is $\left|G_{i(p-1)}^{(n)}\right| \geq 1-T_{i(p-1)}$.

Thus, on the basis of the above estimates for $\left|G_{i(p-1)}^{(n)}\right|$, we are convinced that the third inequality in (13) is valid.

Now, for convenience, we set

$$
\begin{aligned}
& R_{i(k), j}^{(n)}=\cos \alpha_{i(k)}+\sum_{1 \leq i_{k+1} \leq i_{k}, i_{k+1} \neq j} \frac{x_{i(k+1)} u_{i(k+1)}^{(n)}+y_{i(k+1)} v_{i(k+1)}^{(n)}}{\left|G_{i(k+1)}^{(n)}\right|^{2}}, \\
& Q_{i(k), j}^{(n)}=\sin \alpha_{i(k)}+\sum_{1 \leq i_{k+1} \leq i_{k}, i_{k+1} \neq j} \frac{y_{i(k+1)} u_{i(k+1)}^{(n)}-x_{i(k+1)} v_{i(k+1)}^{(n)}}{\left|G_{i(k+1)}^{(n)}\right|^{2}},
\end{aligned}
$$

where $i(k) \in \mathcal{I}, 1 \leq k \leq n-1, n \geq 2$, and $1 \leq j \leq i_{k}$. Then for an arbitrary $1 \leq j \leq i_{k}$ we write

$$
G_{i(k)}^{(n)} e^{i \alpha_{i(k)}}=R_{i(k), j}^{(n)}+i Q_{i(k), j}^{(n)}+\frac{x_{i(k), j}+i y_{i(k), j}}{u_{i(k), j}^{(n)}+i v_{i(k), j}^{(n)}}, i(k) \in \mathcal{I}, 1 \leq k \leq n-1, n \geq 2
$$

where according to $(6)-(7)$

$$
\begin{gathered}
x_{i(k), j}=\operatorname{Re}\left(a_{i(k), j} e^{i\left(\alpha_{i(k)}+\alpha_{i(k), j}\right)}\right), y_{i(k), j}=\operatorname{Im}\left(a_{i(k), j} e^{i\left(\alpha_{i(k)}+\alpha_{i(k), j}\right)}\right), \\
u_{i(k), j}^{(n)}=\operatorname{Re}\left(G_{i(k), j}^{(n)} e^{i \alpha_{i(k), j}}\right), v_{i(k), j}^{(n)}=\operatorname{Im}\left(G_{i(k), j}^{(n)} e^{i \alpha_{i(k), j}}\right) .
\end{gathered}
$$

Hence for all $1 \leq j \leq i_{k}$ the following relations are valid

$$
\begin{gathered}
\left|G_{i(k)}^{(n)}\right|^{2}=\left(R_{i(k), j}^{(n)}\right)^{2}+\left(Q_{i(k), j}^{(n)}\right)^{2}+\frac{\left|a_{i(k), j}\right|^{2}}{\left|G_{i(k), j}^{(n)}\right|^{2}}+2 R_{i(k), j}^{(n)} \frac{x_{i(k), j} u_{i(k), j}^{(n)}+y_{i(k), j} v_{i(k), j}^{(n)}}{\left|G_{i(k), j}^{(n)}\right|^{2}}+ \\
+2 Q_{i(k), j}^{(n)} \frac{y_{i(k), j} u_{i(k), j}^{(n)}-x_{i(k), j} v_{i(k), j}^{(n)}}{\left|G_{i(k), j}^{(n)}\right|^{2}}, i(k) \in \mathcal{I}, 1 \leq k \leq n-1, n \geq 2 .
\end{gathered}
$$


Additionally we set

$$
M_{i(k), j}=\left(\left(1-T_{i(k)}\right)^{2}-\left(t_{i(k), j}\right)^{2}\right) \min \left\{\sin ^{2} \alpha_{i(k)}, \cos ^{2} \alpha_{i(k)}\right\}+\sin ^{2} \alpha_{i(k)} \cos ^{2} \alpha_{i(k)},
$$

where $i(k) \in \mathcal{I}$ and $1 \leq j \leq i_{k}$.

Theorem 2. Let there exist positive constants $d, l$ and $L$ such that the elements of the BCF (1) satisfy the inequalities (11), (12) and for all $i(k) \in \mathcal{I}$

$$
d \leq\left(\mu_{i(k), j}\right)^{2} M_{i(k), j}, 1 \leq j \leq i_{k},\left|a_{i(1)}\right| \leq l \text { and }\left|a_{i(k)}\right| \leq L, k \geq 2,
$$

where $\mu_{i(k), j}$ and $M_{i(k), j}, i(k) \in \mathcal{I}, 1 \leq j \leq i_{k}$, are defined by (9) and (17), respectively. Then the $B C F$ (1) converges to a finite value $f$, and if $f_{n}$ denotes the $n$-th approximant, then

$$
\left|f-f_{n}\right| \leq C_{N+n}^{N-1} \frac{l L^{n}}{d_{0}\left(L^{2}+d\right)^{n / 2}}, n \geq 1
$$

where $d_{0}=\min _{1 \leq i_{1} \leq N} \mu_{i(1)}$.

Proof. Since the conditions of Lemma 1 are satisfied, the relation (13) is valid. From inequalities (13) and (18) it follows that $\left|a_{i(k)}\right| /\left|G_{i(k)}^{(n)}\right| \leq L / \mu_{i(k)}$ for all $i(k) \in \mathcal{I}, 1 \leq k \leq n$, and $n \in \mathbb{N}$.

Let $n$ be an arbitrary integer number, and let $n \geq 2$. Using inequalities (11)-(13), (18), for each multiindex $i(k) \in \mathcal{I}, 1 \leq k \leq n-1$, and for each index $1 \leq j \leq i_{k}$ we estimate the following value $\left|a_{i(k), j}\right|^{2} /\left|G_{i(k)}^{(n)} G_{i(k), j}^{(n)}\right|^{2}$.

Initially for the values $R_{i(k), j}^{(n)}$ and $Q_{i(k), j}^{(n)}$ defined by (14) and (15) respectively, for an arbitrary multiindex $i(k) \in \mathcal{I}, 1 \leq k \leq n-1$, and an arbitrary index $1 \leq j \leq i_{k}$ we have

$$
\begin{aligned}
& R_{i(k), j}^{(n)} \geq \cos \alpha_{i(k)}+\sum_{1 \leq i_{k+1} \leq i_{k}, i_{k+1} \neq j} \frac{x_{i(k+1)} u_{i(k+1)}^{(n)}}{\left|G_{i(k+1)}^{(n)}\right|^{2}} \geq \cos \alpha_{i(k)}+\sum_{i_{k+1} \in \mathcal{I}_{i(k)}^{(2)} \backslash\{j\}} \frac{x_{i(k+1)} u_{i(k+1)}^{(n)}}{\left|G_{i(k+1)}^{(n)}\right|^{2}} \geq \\
& \geq \cos \alpha_{i(k)}-\cos \alpha_{i(k)} \sum_{i_{k+1} \in \mathcal{I}_{i(k)}^{(2)} \backslash\{j\}} \frac{t_{i(k+1)}}{\left|G_{i(k+1)}^{(n)}\right|} \mu_{i(k+1)}=\left(1-S_{i(k), j}^{(2)}\right) \cos \alpha_{i(k)}, \\
& Q_{i(k), j}^{(n)} \geq \sin \alpha_{i(k)}-\sum_{i_{k+1} \in \mathcal{I}_{i(k)}^{(1)} \backslash\{j\}} \frac{x_{i(k+1)} v_{i(k+1)}^{(n)}}{\left|G_{i(k+1)}^{(n)}\right|^{2}} \geq\left(1-S_{i(k), j}^{(1)}\right) \sin \alpha_{i(k)},
\end{aligned}
$$

where $S_{i(k), j}^{(r)}=\sum_{i_{k+1} \in \mathcal{I}_{i(k)}^{(r)} \backslash\{j\}} t_{i(k+1)}, r=1,2$.

Then, for the value $\left|G_{i(k)}^{(n)}\right|^{2}$ we obtain from (16)

$$
\left|G_{i(k)}^{(n)}\right|^{2} \geq\left(R_{i(k), j}^{(n)}\right)^{2}+2 R_{i(k), j}^{(n)} \frac{x_{i(k), j} u_{i(k), j}^{(n)}}{\left|G_{i(k), j}^{(n)}\right|^{2}}+\left(Q_{i(k), j}^{(n)}\right)^{2}-2 Q_{i(k), j}^{(n)} \frac{x_{i(k), j} v_{i(k), j}^{(n)}}{\left|G_{i(k), j}^{(n)}\right|^{2}}+\frac{\left|a_{i(k), j}\right|^{2}}{\left|G_{i(k), j}^{(n)}\right|^{2}} .
$$

Next, we consider separately the cases $x_{i(k), j} \geq 0$ and $x_{i(k), j}<0$. Taking into account that $T_{i(k)}=S_{i(k), j}^{(1)}+S_{i(k), j}^{(2)}+t_{i(k), j}$ for all $1 \leq j \leq i_{k}$, in the first case we have

$$
\left|G_{i(k)}^{(n)}\right|^{2}-\frac{\left|a_{i(k), j}\right|^{2}}{\left|G_{i(k), j}^{(n)}\right|^{2}} \geq\left(R_{i(k), j}^{(n)}\right)^{2}+Q_{i(k), j}^{(n)}\left(Q_{i(k), j}^{(n)}-2 \frac{x_{i(k), j} v_{i(k), j}^{(n)}}{\left|G_{i(k), j}^{(n)}\right|^{2}}\right) \geq
$$




$$
\begin{gathered}
\geq\left(R_{i(k), j}^{(n)}\right)^{2}+Q_{i(k), j}^{(n)}\left(\left(1-S_{i(k), j}^{(1)}\right) \sin \alpha_{i(k)}-2 \frac{t_{i(k), j}}{\left|G_{i(k), j}^{(n)}\right|} \mu_{i(k), j} \sin \alpha_{i(k)}\right) \geq \\
\geq\left(1-S_{i(k), j}^{(2)}\right)^{2} \cos ^{2} \alpha_{i(k)}+\left(1-S_{i(k), j}^{(1)}\right)\left(1-S_{i(k), j}^{(1)}-2 t_{i(k), j}\right) \sin ^{2} \alpha_{i(k)}= \\
=\left(1-S_{i(k), j}^{(2)}\right)^{2}\left(1+\cos 2 \alpha_{i(k)}\right) / 2+\left(1-S_{i(k), j}^{(1)}\right)\left(1-S_{i(k), j}^{(1)}-2 t_{i(k), j}\right)\left(1-\cos 2 \alpha_{i(k)}\right) / 2 .
\end{gathered}
$$

Let $C_{i(k), j}=\left(1-S_{i(k), j}^{(1)}\right)\left(1-S_{i(k), j}^{(1)}-2 t_{i(k), j}\right)+\left(1-S_{i(k), j}^{(2)}\right)^{2}, D_{i(k), j}=\left(1-S_{i(k), j}^{(2)}\right)^{2}-(1-$ $\left.S_{i(k), j}^{(1)}\right)\left(1-S_{i(k), j}^{(1)}-2 t_{i(k), j}\right)$. Then

$$
\begin{gathered}
C_{i(k), j}=\left(1-T_{i(k)}+t_{i(k), j}+S_{i(k), j}^{(2)}\right)\left(1-T_{i(k)}-t_{i(k), j}+S_{i(k), j}^{(2)}\right)+\left(1-S_{i(k), j}^{(2)}\right)^{2}= \\
=\left(1-T_{i(k)}+S_{i(k), j}^{(2)}\right)^{2}-\left(t_{i(k), j}\right)^{2}+\left(1-S_{i(k), j}^{(2)}\right)^{2}= \\
=2\left(S_{i(k), j}^{(2)}\right)^{2}-2 S_{i(k), j}^{(2)}+1+\left(1-T_{i(k)}\right)^{2}+2 S_{i(k), j}^{(2)}\left(1-T_{i(k)}\right)-\left(t_{i(k), j}\right)^{2}, \\
D_{i(k), j}=\left(1-S_{i(k), j}^{(2)}\right)^{2}-\left(1-T_{i(k)}+t_{i(k), j}+S_{i(k)}^{(2)}\right)\left(1-T_{i(k)}-t_{i(k), j}+S_{i(k), j}^{(2)}\right)= \\
=\left(1-S_{i(k), j}^{(2)}\right)^{2}-\left(1-T_{i(k)}+S_{i(k), j}^{(2)}\right)^{2}+\left(t_{i(k), j}\right)^{2}= \\
=1-\left(1-T_{i(k)}\right)^{2}-2 S_{i(k), j}^{(2)}\left(1-T_{i(k)}\right)-2 S_{i(k), j}^{(2)}+\left(t_{i(k), j}\right)^{2}
\end{gathered}
$$

and therefore

$$
\begin{gathered}
C_{i(k), j}+D_{i(k), j} \cos 2 \alpha_{i(k)}=2\left(S_{i(k), j}^{(2)}\right)^{2}+1+\cos 2 \alpha_{i(k)}-2\left(1+\cos 2 \alpha_{i(k)}\right) S_{i(k), j}^{(2)}+ \\
+\left(\left(1-T_{i(k)}\right)^{2}-\left(t_{i(k), j}\right)^{2}\right)\left(1-\cos 2 \alpha_{i(k)}\right)+2 S_{i(k), j}^{(2)}\left(1-T_{i(k)}\right)\left(1-\cos 2 \alpha_{i(k)}\right) \geq \\
\geq\left(\left(1-T_{i(k)}\right)^{2}-\left(t_{i(k), j}\right)^{2}\right)\left(1-\cos 2 \alpha_{i(k)}\right)+2\left(S_{i(k), j}^{(2)}-\left(1+\cos 2 \alpha_{i(k)}\right) / 2\right)^{2}+1+\cos 2 \alpha_{i(k)}- \\
-\left(1+\cos 2 \alpha_{i(k)}\right)^{2} / 2 \geq\left(\left(1-T_{i(k)}\right)^{2}-\left(t_{i(k), j}\right)^{2}\right) 2 \sin ^{2} \alpha_{i(k)}+\left(\sin ^{2} 2 \alpha_{i(k)}\right) / 2 .
\end{gathered}
$$

In the second case, as $x_{i(k), j}<0$ we have

$$
\begin{gathered}
\left|G_{i(k)}^{(n)}\right|^{2}-\frac{\left|a_{i(k), j}\right|^{2}}{\left|G_{i(k), j}^{(n)}\right|^{2}} \geq\left(Q_{i(k), j}^{(n)}\right)^{2}+R_{i(k), j}^{(n)}\left(R_{i(k), j}^{(n)}+2 \frac{x_{i(k), j} v_{i(k), j}^{(n)}}{\left|G_{i(k), j}^{(n)}\right|^{2}}\right) \geq \\
\geq\left(Q_{i(k), j}^{(n)}\right)^{2}+R_{i(k), j}^{(n)}\left(\left(1-S_{i(k), j}^{(2)}\right) \cos \alpha_{i(k)}-2 \frac{t_{i(k), j}}{\left|G_{i(k), j}^{(n)}\right|} \mu_{i(k), j} \sin \alpha_{i(k)}\right) \geq \\
\geq\left(1-S_{i(k), j}^{(1)}\right)^{2} \sin ^{2} \alpha_{i(k)}+\left(1-S_{i(k), j}^{(2)}\right)\left(1-S_{i(k), j}^{(2)}-2 t_{i(k), j}\right) \cos ^{2} \alpha_{i(k)}= \\
=\left(1-S_{i(k), j}^{(1)}\right)^{2}\left(1-\cos 2 \alpha_{i(k)}\right) / 2+\left(1-S_{i(k), j}^{(2)}\right)\left(1-S_{i(k), j}^{(2)}-2 t_{i(k), j}\right)\left(1+\cos 2 \alpha_{i(k)}\right) / 2 .
\end{gathered}
$$

Repeating arguments from the case $x_{i(k) j} \geq 0$, we obtain

$$
\begin{gathered}
E_{i(k), j}-F_{i(k), j} \cos 2 \alpha_{i(k)} \geq\left(\left(1-T_{i(k)}\right)^{2}-\left(t_{i(k), j}\right)^{2}\right)\left(1+\cos 2 \alpha_{i(k)}\right)+ \\
+1-\cos 2 \alpha_{i(k)}-\left(1-\cos 2 \alpha_{i(k)}\right)^{2} / 2=\left(\left(1-T_{i(k)}\right)^{2}-\left(t_{i(k), j}\right)^{2}\right) 2 \cos ^{2} \alpha_{i(k)}+\left(\sin ^{2} 2 \alpha_{i(k)}\right) / 2,
\end{gathered}
$$

where $E_{i(k), j}=\left(1-S_{i(k), j}^{(1)}\right)^{2}+\left(1-S_{i(k), j}^{(2)}\right)\left(1-S_{i(k), j}^{(2)}-2 t_{i(k), j}\right), F_{i(k), j}=\left(1-S_{i(k), j}^{(1)}\right)^{2}-(1-$ $\left.S_{i(k), j}^{(2)}\right)\left(1-S_{i(k), j}^{(2)}-2 t_{i(k), j}\right)$.

Thus, in both cases, the following inequality holds $\left|G_{i(k)}^{(n)}\right|^{2} \geq\left|a_{i(k), j}\right|^{2} /\left|G_{i(k), j}^{(n)}\right|^{2}+M_{i(k), j}$. 
Finally, for the values $\left|a_{i(k), j}\right|^{2} /\left|G_{i(k)}^{(n)} G_{i(k), j}^{(n)}\right|^{2}$ we have

$$
\frac{\left|a_{i(k), j}\right|^{2}}{\left|G_{i(k)}^{(n)} G_{i(k), j}^{(n)}\right|^{2}} \leq \frac{\left|a_{i(k), j}\right|^{2} /\left|G_{i(k), j}^{(n)}\right|^{2}}{M_{i(k), j}+\left|a_{i(k), j}\right|^{2} /\left|G_{i(k), j}^{(n)}\right|^{2}} \leq \frac{L^{2}}{M_{i(k), j}\left(\mu_{i(k), j}\right)^{2}+L^{2}} \leq \frac{L^{2}}{d+L^{2}}
$$

This means that the fundamental inequalities (3) and (4) are valid, where $M=l / d_{0}$ and $\rho_{n}=L /\left(L^{2}+d\right)^{1 / 2}, n \geq 2$. Therefore, on the basis of Theorem 1 and its remark, we obtain the statement of this theorem.

We note, that for certain possible values of numbers of sequences $\left\{t_{i(k)}\right\}_{i(k) \in \mathcal{I}, k \geq 2}$ and $\left\{\alpha_{i(k)}\right\}_{i(k) \in \mathcal{I}}$ from Theorem 2, we can obtain some simple and constructive conditions for error bounds for BCF (1).

For example, let

$$
t_{i(k)}=1 /\left(i_{k-1}+1\right) \text { for all } i(k) \in \mathcal{I}, k \geq 2 \text { and } \alpha_{i(k)}=\alpha_{i_{k}} \text { for all } i(k) \in \mathcal{I} .
$$

Then from Theorem 2 we have a corollary.

Corollary 1. Let there exist positive constants $l$ and $L$ such that the elements of the $B C F$ (1) satisfy the inequalities $\left|a_{i(1)}\right| \leq l$ for all $i(1) \in \mathcal{I}$ and $\left|a_{i(k)}\right| \leq L$,

$$
-\mu_{i_{k}} \cos \alpha_{i_{k-1}} \leq\left(i_{k-1}+1\right) \operatorname{Re}\left(a_{i(k)} e^{i\left(\alpha_{i_{k-1}}+\alpha_{i_{k}}\right)}\right) \leq \mu_{i_{k}} \sin \alpha_{i_{k-1}}, \operatorname{Im}\left(a_{i(k)} e^{i\left(\alpha_{i_{k-1}}+\alpha_{i_{k}}\right)}\right) \geq 0
$$

for all $i(k) \in \mathcal{I}$ and $k \geq 2$, where

$$
\mu_{k}=\max \left\{\left(\cos ^{2} \alpha_{k}+\frac{1}{(k+1)^{2}}\right)^{1 / 2} \sin \alpha_{k}, \frac{1}{k+1}, \frac{k+2}{2 k+2} \sin 2 \alpha_{k}\right\},
$$

$\alpha_{k} \in(0 ; \pi / 2), 1 \leq k \leq N$. Then the BCF (1) converges to a finite value $f$ and the inequalities (19), where

$$
d_{0}=\min _{1 \leq k \leq N} \mu_{k} \text { and } d=\min _{i(2) \in \mathcal{I}} \frac{\left(\mu_{i_{2}}\right)^{2} \sin ^{2} 2 \alpha_{i_{1}}}{4}
$$

are valid.

Now, let for all $i(k) \in \mathcal{I}$ and $k \geq 2$ the values $y_{i(k)}$ defined in (6) are nonpositive. Then by analogy to proof of Lemma 1, we obtain the following lemma.

Lemma 2. Let the elements of the BCF (1) satisfy the inequalities

$$
-t_{i(k)} \nu_{i(k)} \cos \alpha_{i(k-1)} \leq x_{i(k)} \leq t_{i(k)} \nu_{i(k)}\left|\sin \alpha_{i(k-1)}\right|, y_{i(k)} \leq 0 \text { for all } i(k) \in \mathcal{I}, k \geq 2,
$$

where $x_{i(k)}, y_{i(k)}, i(k) \in \mathcal{I}, k \geq 2$, are defined by $(6)$;

$$
\nu_{i(k)}=\max \left\{\left(\cos ^{2} \alpha_{i(k)}+\left(1-T_{i(k)}\right)^{2}\right)^{1 / 2}\left|\sin \alpha_{i(k)}\right|, 1-T_{i(k)},\left(1-T_{i(k)} / 2\right)\left|\sin 2 \alpha_{i(k)}\right|\right\} ;
$$

$t_{i(k)}, k \geq 2$, and $\alpha_{i(k)}, i(k) \in \mathcal{I}$, are real numbers such that

$$
t_{i(k)}>0, k \geq 2, T_{i(k)} \leq 1,-\pi / 2<\alpha_{i(k)}<0, i(k) \in \mathcal{I},
$$


where $T_{i(k)}, i(k) \in \mathcal{I}$, are defined by (8). Then for all $i(k) \in \mathcal{I}, 1 \leq k \leq n$, and $n \in \mathbb{N}$ the following inequalities are valid

$$
u_{i(k)}^{(n)} \geq\left(1-T_{i(k)}^{(2)}\right) \cos \alpha_{i(k)}, v_{i(k)}^{(n)} \leq\left(1-T_{i(k)}^{(1)}\right) \sin \alpha_{i(k)} \text { and }\left|G_{i(k)}^{(n)}\right| \geq \nu_{i(k)}>0,
$$

where $G_{i(k)}^{(n)}$ and $u_{i(k)}^{(n)}, v_{i(k)}^{(n)}, i(k) \in \mathcal{I}, 1 \leq k \leq n, n \in \mathbb{N}$, are defined by (2) and (7), respectively; $T_{i(k)}^{(r)}=\sum_{i_{k+1} \in \mathcal{I}_{i(k)}^{(r)}} t_{i(k+1)}, i(k) \in \mathcal{I}, r=1,2 ; \mathcal{I}_{i(k)}^{(r)}, i(k) \in \mathcal{I}, r=1,2$, are defined by (10).

Using Lemma 2, by analogy to the proof of Theorem 2 yield the following theorem.

Theorem 3. Let there exist positive constants $d, l$ and $L$ such that the elements of the BCF (1) satisfy the inequalities (23), (25) and for all $i(k) \in \mathcal{I}$

$$
d \leq\left(\nu_{i(k), j}\right)^{2} M_{i(k), j}, 1 \leq j \leq i_{k},\left|a_{i(1)}\right| \leq l \text { and }\left|a_{i(k)}\right| \leq L, k \geq 2,
$$

where $M_{i(k), j}$ and $\nu_{i(k), j}, i(k) \in \mathcal{I}, 1 \leq j \leq i_{k}$, are defined by (17) and (24), respectively. Then the $B C F$ (1) converges to a finite value $f$ and the inequalities (19), where $d_{0}=\min _{1 \leq i_{1} \leq N} \nu_{i(1)}$, are valid.

In view of (20), Theorem 3 imply the following corollary.

Corollary 2. Let there exist positive constants $l$ and $L$ such that the elements of the BCF (1) satisfy the inequalities $\left|a_{i(1)}\right| \leq l$ for all $i(1) \in \mathcal{I}$ and $\left|a_{i(k)}\right| \leq L$,

$$
-\nu_{i_{k}} \cos \alpha_{i_{k-1}} \leq\left(i_{k-1}+1\right) \operatorname{Re}\left(a_{i(k)} e^{i\left(\alpha_{i_{k-1}}+\alpha_{i_{k}}\right)}\right) \leq \nu_{i_{k}}\left|\sin \alpha_{i_{k-1}}\right|, \operatorname{Im}\left(a_{i(k)} e^{i\left(\alpha_{i_{k-1}}+\alpha_{i_{k}}\right)}\right) \leq 0
$$

for all $i(k) \in \mathcal{I}$ and $k \geq 2$, where

$$
\nu_{k}=\max \left\{\left(\cos ^{2} \alpha_{k}+\frac{1}{(k+1)^{2}}\right)^{1 / 2}\left|\sin \alpha_{k}\right|, \frac{1}{k+1}, \frac{k+2}{2 k+2}\left|\sin 2 \alpha_{k}\right|\right\},
$$

$\alpha_{k} \in(-\pi / 2 ; 0), 1 \leq k \leq N$. Then the BCF (1) converges to a finite value $f$ and the inequalities (19) are valid, where

$$
d_{0}=\min _{1 \leq k \leq N} \nu_{k} \text { and } d=\min _{i(2) \in \mathcal{I}} \frac{\left(\nu_{i_{2}}\right)^{2} \sin ^{2} 2 \alpha_{i_{1}}}{4} .
$$

3. Applications. Perhaps the greatest value of the results of Section 2 is associated with deriving error bounds for BCF (1) whose elements $a_{i(k)}, i(k) \in \mathcal{I}$, are functions of one or several complex variable. To illustrate this use we shall give applications to multidimensional $S-, A-, J$-fractions with independent variables. Expansions of certain analytic functions in some of these classes of continued fractions can be found in $[12,14,16,18,19,20]$. BCF of the form

$$
\sum_{i_{1}=1}^{N} \frac{c_{i(1)} z_{i_{1}}}{1}+\sum_{i_{2}=1}^{i_{1}} \frac{c_{i(2)} z_{i_{2}}}{1}+\sum_{i_{3}=1}^{i_{2}} \frac{c_{i(3)} z_{i_{3}}}{1}+\cdots
$$

where $c_{i(k)}>0$ for all $i(k) \in \mathcal{I}, \mathbf{z}=\left(z_{1}, z_{2}, \ldots, z_{n}\right) \in \mathbb{C}^{N}$, are the so-called multidimensional $S$-fractions with independent variables. 
Example 1. Let $r>0$ be given. Let $\left\{c_{i(k)}\right\}_{i(k) \in \mathcal{I}}$ be a sequence of positive numbers satisfying $c_{i(1)} \leq r / 2$ for all $i(1) \in \mathcal{I}$ and $c_{i(k)} \leq r /\left(i_{k-1}+1\right)$ for all $i(k) \in \mathcal{I}, k \geq 2$. Let $g_{n}(\mathbf{z})$ denote the $n$-th approximant of the multidimensional $S$-fraction with independent variables (28). Then for all $z_{k}=\left|z_{k}\right| e^{i \varphi_{k}}, 1 \leq k \leq N$, such that $\left|\varphi_{k}\right|=\alpha$ and $\left|z_{k}\right| \leq\left(\mu_{k} \sin \alpha\right) /\left(r\left|\cos \left(2 \alpha+\varphi_{k}\right)\right|\right)$, $1 \leq k \leq N$, where $\alpha \in(\pi / 6 ; \pi / 4]$ and

$$
\mu_{k}=\max \left\{\left(\cos ^{2} \alpha+\frac{1}{(k+1)^{2}}\right)^{1 / 2} \sin \alpha, \frac{1}{k+1}, \frac{k+2}{2 k+2} \sin 2 \alpha\right\}, 1 \leq k \leq N,
$$

there exists the limit $g(\mathbf{z})=\lim _{n \rightarrow \infty} g_{n}(\mathbf{z})$ and

$$
\left|g(\mathbf{z})-g_{n}(\mathbf{z})\right| \leq C_{N+n}^{N-1} L^{n+1} /\left(d_{0}\left(L^{2}+d\right)^{n / 2}\right), n \geq 1,
$$

where $d_{0}=\min _{1 \leq k \leq N} \mu_{k}, d=\left(d_{0}^{2} / 4\right) \sin ^{2} 2 \alpha, L=(\mu \sin \alpha) /(2|\cos 3 \alpha|), \mu=\max _{1 \leq k \leq N} \mu_{k}$.

This example follows from Corollary 1 by taking $a_{i(k)}=c_{i(k)} z_{i_{k}}, i(k) \in \mathcal{I}$, and $\alpha_{k}=\alpha$, $1 \leq k \leq N$.

Multidimensional $A$-fractions with independent variables are of the form

$$
\sum_{i_{1}=1}^{N} \frac{p_{i(1)} z_{i_{1}}}{1}+\sum_{i_{2}=1}^{i_{1}} \frac{(-1)^{\delta_{i_{1}, i_{2}}} p_{i(2)} z_{i_{1}} z_{i_{2}}}{1}+\sum_{i_{3}=1}^{i_{2}} \frac{(-1)^{\delta_{i_{2}, i_{3}}} p_{i(3)} z_{i_{2}} z_{i_{3}}}{1}+\ldots
$$

where $p_{i(k)} \in \mathbb{C} \backslash\{0\}$ for all $i(k) \in \mathcal{I}, \delta_{i, j}$ is the Kronecker delta.

Example 2. Let $r>0$ be given. Let $\left\{p_{i(k)}\right\}_{i(k) \in \mathcal{I}}$ be a sequence of numbers satisfying $\left|p_{i(1)}\right| \leq r^{1 / 2}$ for all $i(1) \in \mathcal{I}$ and $-r /\left(i_{k-1}+1\right) \leq(-1)^{\delta_{i_{k-1}, i_{k}}} p_{i(k)}<0$ for all $i(k) \in \mathcal{I}, k \geq 2$.

Let $h_{n}(\mathbf{z})$ denote the $n$-th approximant of the multidimensional $A$-fraction with independent variables (29). Then for all $z_{k}=\left|z_{k}\right| e^{i \varphi_{k}}, 1 \leq k \leq N$, such that

$$
\varphi_{i_{1}}=-2 \alpha_{i_{1}} \text { for all } i_{1} \in \mathcal{I} \text { and }\left|z_{i_{1}} z_{i_{2}}\right| \leq \frac{d_{0} \min \left\{\left|\sin \alpha_{i_{1}}\right|, \cos \alpha_{i_{1}}\right\}}{r\left|\cos \left(\alpha_{i_{1}}+\alpha_{i_{2}}\right)\right|} \text { for all } i(2) \in \mathcal{I},
$$

where $\alpha_{i_{1}} \in(-\pi / 2 ; 0), i_{1} \in \mathcal{I} ; \alpha_{i_{1}}+\alpha_{i_{2}} \neq-\pi / 2, i(2) \in \mathcal{I} ; d_{0}$ is defined as in (27); there exists the limit $h(\mathbf{z})=\lim _{n \rightarrow \infty} h_{n}(\mathbf{z})$ and

$$
\left|h(\mathbf{z})-h_{n}(\mathbf{z})\right| \leq C_{N+n}^{N-1} l L^{n} /\left(d_{0}^{1 / 2}\left(L^{2}+d\right)^{n / 2}\right), n \geq 1,
$$

where $d$ is defined as in (27),

$$
l=\left(\max _{1 \leq i_{1} \leq N} \frac{\min \left\{\left|\sin \alpha_{i_{1}}\right|, \cos \alpha_{i_{1}}\right\}}{\left|\cos 2 \alpha_{i_{1}}\right|}\right)^{1 / 2}, L=\frac{d_{0}}{2} \max _{1 \leq i_{1} \leq N} \min _{1 \leq i_{2} \leq i_{1}} \frac{\min \left\{\left|\sin \alpha_{i_{1}}\right|, \cos \alpha_{i_{1}}\right\}}{\left|\cos \left(\alpha_{i_{1}}+\alpha_{i_{2}}\right)\right|} .
$$

This result follows immediately from Corollary 2 by taking $a_{i(1)}=p_{i(1)} z_{i_{1}}, 1 \leq i_{1} \leq N$, $a_{i(k)}=(-1)^{\delta_{i_{k-1}, i_{k}}} p_{i(k)} z_{i_{k-1}} z_{i_{k}}, i(k) \in \mathcal{I}, k \geq 2$.

Multidimensional $J$-fractions with independent variables are of the form

$$
\sum_{i_{1}=1}^{N} \frac{q_{i(1)}}{z_{i_{1}}}+\sum_{i_{2}=1}^{i_{1}} \frac{(-1)^{\delta_{i_{1}, i_{2}}} q_{i(2)}}{z_{i_{2}}}+\sum_{i_{3}=1}^{i_{2}} \frac{(-1)^{\delta_{i_{2}, i_{3}}} q_{i(3)}}{z_{i_{3}}}+\cdots,
$$

where $q_{i(k)} \in \mathbb{C} \backslash\{0\}$ for all $i(k) \in \mathcal{I}$. 
Example 3. Let $r>0$ be given. Let $\left\{q_{i(k)}\right\}_{i(k) \in \mathcal{I}}$ be a sequence of numbers satisfying

$\left|q_{i(1)}\right| \leq r^{1 / 2}$ for all $i(1) \in \mathcal{I}$ and $-r /\left(i_{k-1}+1\right) \leq(-1)^{\delta_{i_{k-1}, i_{k}}} q_{i(k)}<0$ for all $i(k) \in \mathcal{I}, k \geq 2$.

Let $f_{n}(\mathbf{z})$ denote the $n$-th approximant of the multidimensional $J$-fraction with independent variables (30). Then for all $z_{k}=\left|z_{k}\right| e^{i \varphi_{k}}, 1 \leq k \leq N$, such that

$$
\varphi_{i_{1}}=2 \alpha_{i_{1}} \text { for all } i_{1} \in \mathcal{I} \text { and }\left|z_{i_{1}} z_{i_{2}}\right| \geq \frac{r\left|\cos \left(\alpha_{i_{1}}+\alpha_{i_{2}}\right)\right|}{d_{0} \min \left\{\sin \alpha_{i_{1}}, \cos \alpha_{i_{1}}\right\}} \text { for all } i(2) \in \mathcal{I} \text {, }
$$

where $\alpha_{i_{1}} \in(0 ; \pi / 2), i_{1} \in \mathcal{I} ; \alpha_{i_{1}}+\alpha_{i_{2}} \neq \pi / 2, i(2) \in \mathcal{I} ; d_{0}$ is defined as in (22); there exists the limit $f(\mathbf{z})=\lim _{n \rightarrow \infty} f_{n}(\mathbf{z})$ and

$$
\left|f(\mathbf{z})-f_{n}(\mathbf{z})\right| \leq C_{N+n}^{N-1} l L^{n} /\left(d_{0}^{1 / 2}\left(L^{2}+d\right)^{n / 2}\right), n \geq 1,
$$

where $d$ is defined as in (22),

$$
l=\left(\max _{1 \leq i_{1} \leq N} \frac{\min \left\{\sin \alpha_{i_{1}}, \cos \alpha_{i_{1}}\right\}}{\left|\cos 2 \alpha_{i_{1}}\right|}\right)^{1 / 2}, L=\frac{d_{0}}{2} \max _{1 \leq i_{1} \leq N} \min _{1 \leq i_{2} \leq i_{1}} \frac{\min \left\{\sin \alpha_{i_{1}}, \cos \alpha_{i_{1}}\right\}}{\left|\cos \left(\alpha_{i_{1}}+\alpha_{i_{2}}\right)\right|} .
$$

This assertion follows from Corollary 1 applied to the equivalent BCF

$$
\sum_{i_{1}=1}^{N} \frac{q_{i(1)} \xi_{i_{1}}}{1}+\sum_{i_{2}=1}^{i_{1}} \frac{(-1)^{\delta_{i_{1}, i_{2}}} q_{i(2)} \xi_{i_{1}} \xi_{i_{2}}}{1}+\sum_{i_{3}=1}^{i_{2}} \frac{(-1)^{\delta_{i_{2}, i_{3}}} q_{i(3)} \xi_{i_{1}} \xi_{i_{3}}}{1}+\ldots
$$

where $\xi_{k}=1 / z_{k}, 1 \leq k \leq N$. Thus in Corollary 1 we set $a_{i(1)}=q_{i(1)} \xi_{i_{1}}, 1 \leq i_{1} \leq N$, $a_{i(k)}=(-1)^{\delta_{i_{k-1}, i_{k}}} q_{i(k)} \xi_{i_{k-1}} \xi_{i_{k}}, i(k) \in \mathcal{I}, k \geq 2$.

4. Conclusions. The paper gives estimates of the rate of convergence of BCF (1) whose elements belong to rectangular regions unlike before known circular and angular regions. The method proposed here for the study of BCF (1) can be used to study their stability. Still there is a problem that remains open. How to take the real numbers of sequences $\left\{t_{i(k)}\right\}_{i(k) \in \mathcal{I}, k \geq 2}$ and $\left\{\alpha_{i(k)}\right\}_{i(k) \in \mathcal{I}}$ to obtain the widest regions of variables for functional branched continued fractions with independent variables.

\section{REFERENCES}

1. T.M. Antonova, Speed of convergence of branched continued fractions of the special form, Volynskyi Mat. Visnyk, 6 (1999), 5-11. (in Ukrainian)

2. T.M. Antonova, D.I. Bodnar, Convergence domains for branched continued fractions of the special form, Approx. Theory and its Appl.: Proc. Inst. Math. NAS Ukr., 31 (2000), 19-32. (in Ukrainian)

3. T.M. Antonova, M.V. Dmytryshyn, S.M. Vozna, Some properties of approximants for branched continued fractions of the special form with positive and alternating-sign partial numerators, Carpathian Math. Publ., 10 (2018), №1, 3-13. doi: 10.15330/cmp.10.1.3-13

4. T.M. Antonova, R.I. Dmytryshyn, Truncation error bounds for branched continued fraction $\sum_{i_{1}=1}^{N} \frac{a_{i(1)}}{1}+\sum_{i_{2}=1}^{i_{1}} \frac{a_{i(2)}}{1}+\sum_{i_{3}=1}^{i_{2}} \frac{a_{i(3)}}{1}+\cdots$, Ukr. Mat. Zhurn., 72 (2020), №7, 877-885. (in Ukrainian) doi: $10.37863 /$ umzh.v72i 7.2342 
5. T.M. Antonova, O.M. Sus', On some sequences of the sets of uniform convergence for two-dimensional continued fractions, Mat. method. and fiz.-mech. polya, 58 (2015), №1, 47-56. (in Ukrainian); Engl. transl.: J. Math. Sci., 222 (2017), №1, 56-69. doi: 10.1007/s10958-017-3282-y

6. T.M. Antonova, S.M. Vozna, On one analogue of the method of fundamental inequalities for research of branched continued fractions of the special form, Visnyc Lviv Polytech. Ntl. Univ. Ser. Phys. and Math. Sci., 871 (2017), 5-12. (in Ukrainian)

7. O.E. Baran, Some domains convergence of branched continued fractions of the special form, Carpathian Math. Publ., 5 (2013), №1, 4-13. (in Ukrainian) doi: 10.15330/cmp.5.1.4-13

8. I.B. Bilanyk, A truncation error bound for some branched continued fractions of the special form, Mat. Stud., 52 (2019), №2, 115-123. doi: 10.30970/ms.52.2.115-123

9. I.B. Bilanyk, D.I. Bodnar, L.M. Byak, Representation of a quotient of solutions of a four-term linear recurrence relation in the form of a branched continued fraction, Carpathian Math. Publ., 11 (2019), №1, 33-41. doi: 10.15330/cmp.11.1.33-41

10. D.I. Bodnar, I.B. Bilanyk, On the convergence of branched continued fractions of a special form in angular domains, Mat. method. and fiz.-mech. polya, 60 (2017), №3, 60-69. (in Ukrainian); Engl. transl.: J. Math. Sci. 246 (2020), №2, 188-200. doi: 10.1007/s10958-020-04729-w

11. D.I. Bodnar, Branched continued fractions, Naukova Dumka, Kyiv, 1986. (in Russian)

12. D.I. Bodnar, R.I. Dmytryshyn, Multidimensional associated fractions with independent variables and multiple power series, Ukr. Math. Zhurn., 71 (2019), №3, 325-339. (in Ukrainian); Engl. transl.: Ukrainian Math. J., 71 (2019), №3, 370-386. doi: 10.1007/s11253-019-01652-5

13. M.M. Bubniak, Truncation-error bounds for the 1-periodic branched continued fraction of special form, Carpathian Math. Publ., 5 (2013), №2, 187-195. doi: 10.15330/cmp.5.2.187-195

14. R.I. Dmytryshyn, Associated branched continued fractions with two independent variables, Ukr. Math Zhurn., 66 (2014), №9, 1175-1184. (in Ukrainian); Engl. transl.: Ukrainian Math. J., 66 (2015), №9, 1312-1323. doi: 10.1007/s11253-015-1011-6

15. R.I. Dmytryshyn, Convergence of some branched continued fractions with independent variables, Mat. Stud., 47 (2017), №2, 150-159. doi: 10.15330/ms.47.2.150-159

16. R.I. Dmytryshyn, Multidimensional regular $C$-fraction with independent variables corresponding to formal multiple power series, Proc. Roy. Soc. Edinburgh Sect. A, (2019), 1-18. doi: 10.1017/prm.2019.2

17. R.I. Dmytryshyn, On some of convergence domains of multidimensional $S$-fractions with independent variables, Carpathian Math. Publ., 11 (2019), №1, 54-58. doi: 10.15330/cmp.11.1.54-58

18. R.I. Dmytryshyn, On the expansion of some functions in a two-dimensional g-fraction with independent variables, Mat. method. and fiz.-mech. polya, 53 (2010), №4, 56-69. (in Ukrainian); Engl. transl.: J. Math. Sci., 181 (2012), №3, 320-327. doi: 10.1007/s10958-012-0687-5

19. R.I. Dmytryshyn, The two-dimensional g-fraction with independent variables for double power series, J. Approx. Theory, 164 (2012), №12, 1520-1539. doi: 10.1016/j.jat.2012.09.002

20. R.I. Dmytryshyn, Two-dimensional generalization of the Rutishauser qd-algorithm, Mat. method. and fiz.-mech. polya, 56 (2013), №4, 6-11. (in Ukrainian); Engl. transl.: J. Math. Sci., 208 (2015), №3, 301-309. doi: 10.1007/s10958-015-2447-9

21. W.B. Jones, W.J. Thron, Continued fractions: Analytic theory and applications, Addison-Wesley Pub. Co., Reading, Mass., 1980.

Lviv Polytechnic National University Department of Applied Mathematics Lviv, Ukraine

tamara.m.antonova@lpnu.ua

Vasyl Stefanyk Precarpathian National University

Department of Mathematical and Functional Analysis

Ivano-Frankivsk, Ukraine

dmytryshynr@hotmail.com 